\title{
Application and Normalization of Lettered Words in Chinese Character System
}

\author{
Xinrong Xia \\ Language Studies College, Sichuan Agricultural University \\ Ya'an 625014, Sichuan, China \\ E-mail: xiaxinrong33@163.com
}

Foundation item: one of the achievements of Sichuan Education Departments' fund project of key researches in social science (license number: SA05-002)

\begin{abstract}
Lettered word is a new form of loanword. It is the outcome of information share, science and technology communication as well as cultural integration. The paper classifies lettered words in Chinese character system and points out that English lettered words and Chinese characters differ greatly in their language origin and form. Therefore, lettered words have an unprecedented effect on Chinese lexical system from the aspects of writing form, phonological system, model of meaning expression and word formation. The generally accepted idea is that the use of lettered words should be normalized on the premises of the recognition of lettered words in Chinese lexicon. Finally, the paper puts forward flexible normalization for the absorption of lettered words with an emphasis on the guidance of mass media and dictionary compilation.
\end{abstract}

Keywords: Lettered word, Loanword, Chinese lexical system, Normalization

Language, an outcome of social organization, evolves with social development (Luo, 2004). With the globalization of politics, economy, science and culture, modern Chinese character system can no longer record incessant new things and concepts in a concise, direct and effective way, hence having to turn to loanwords to compensate for its lexical vacancies. As a result, lettered words enter modern Chinese lexical system due to their concision, rich meanings and access to the whole world and play an indispensable role in science and technology field and daily communication. The entry of lettered words into Chinese character system is a beneficial adjustment of a language's recording function made by the language system itself, which makes Chinese more comprehensive and more adapted to the new demands for its recording function by the global communication in the modern information era. Due to the particular importance of lettered words, this paper aims at classifying the lettered words used in Chinese lexical system and exploring the influences of modern Chinese lexical system on them and their normalization.

\section{Types of Lettered Words in Chinese Lexical System}

With lettered words being a special part in Chinese lexical system, the academic circle is trying classifying them (Shi, 2000. Liu, 2002. Liu, 2002. Xue, 2006) but hasn't drawn an accepted conclusion. Seen from the types of the lettered words in the current Chinese lexical system, they are mainly divided into unchanged English lettered words, complex lettered words and self-created Chinese lettered words.

\subsection{Unchanged English Lettered Words}

This type of lettered words refers to those including only letters in a word, which come from two major sources: English abbreviated words and English words.

\subsubsection{English Abbreviated Words}

English abbreviated words are made up of English letters (Liu, 2002) to express as complete meanings as their corresponding full names. They are mainly composed in the following ways:

Acronyms 
1) composed of the initials of all words in a phrase, such as WTO (World Trade Organization), SOS (Save Our Ship), BBC (British Broadcasting Corporation) and DNA (Desoxyribo Nucleic Acid).

2) composed of the initials of all notional words in a phrase, such as GATT (General Agreement on Tariffs and Trade) whose function words (on, and) are omitted, AT\&T ( American Telephone and Telegraph) in which "and" is replaced by "\&".

3) composed of the initial of the former word and the complete latter word, such as E-BANK (Electronic BANK) and A-life (Artificial life).

Abbreviations with representative letters

Here, representative letters are chosen from a word or a phrase to compose an abbreviated word, such as TB (tuberculosis), MSN (MicroSoft Network), Modem (Modulator-Demodulator) and Hi-Fi (High Fidelity).

\subsubsection{Unchanged English Words}

This type refers to those English words or phrases directly used in Chinese without any changes. One part goes into daily expressions, such as copy, Internet, flash, cool and the other goes into jargons or proper nouns, such as Office, Windows 2000 and so on.

\subsection{Complex Lettered Words}

Here this type refers to those words with both English letters and Chinese characters which express complete meanings. Chinese characters play a flexible role in them of definition direction or semantic supplementation. These words are mainly formed in four ways:

First, they can be formed by abbreviated English letters and Chinese characters

1) abbreviated initials, such as e 时代 (electronic era), AA 制 (Algebraic Average) and DINK 家庭 (Double Income, No Kids.).

2) number + representative letters (sometimes the number of common letters shared by several words in a phrase can be used in the form of Arabic numbers), such as 4A 革命 (FA: factory automation, OA: office automation, HA: household automation, AA: agricultural automation), $3 \mathrm{~S}$ 一体化技术 (RS: remote sensing, GIS: geography information system, GPS: global positioning system) and 3A 式服务 (Anytime, Anywhere, Anyway).

3) the initial syllable, such as Esc (Esc=Escape) and Doc (Doc= Document).

Second, the shape of letters can be used to portray things, such as X 型人才 ( talents with interdisciplinary knowledge), $\mathrm{T}$ 恤衫 (T-shirt is in the shape of "T" and "恤” is similar to "shirt" in pronunciation).

Third, some letters expressing order, place and type can be used to form words, such as A 型血, 甲 A, 维 C and X 型染 色体.

Fourth, some words can be formed by complete English words and Chinese characters, such as Call 机 and 服装 SHOW.

\subsection{Self-created Chinese Lettered Words}

This type refers to those lettered words relevant to things with Chinese characteristics, created by Chinese people and initially used in China.

First, lettered words can be formed by Chinese phonetic alphabets due to their similarity to English letters. Some come from the initial of every syllable, such as RMB (Renminbi), and some others come from the initial letter of every word, such as PSC (Putonghua Shuiping Ceshi). Although these lettered words are composed of Chinese onsets, they are pronounced as English letters.

Second, some words are formed by translating things with Chinese characteristics into English and then drawing the initial of each word, such as CCTV (China Central Television) and CBA (Chinese Basketball Association).

Besides the above three types, there are some other ones: lettered words with similar sounds to some English expressions, such as ICQ (a tool on the Internet for chatting, sending messages and documents) which is similar to "I seek you" in English; some formed by English abbreviations and numbers, such as MP3; especially some complicated lettered words related to professional fields, such as Tech-et5500 彩色喷墨绘图仪 and DI-COM3. 0 标准; some even formed by letters, numbers, punctuation marks, symbols and Chinese characters.

\section{Standards for the Use of Lettered Words}

Lettered words have entered modern Chinese lexical system in different ways and forms, providing fresh blood for constant development of this system. However, the random, unordered and irregular use of these words (Liu, 2002) has caused disorder in Chinese lexical system. For the sake of Chinese language, it is quite necessary to confirm the standards for lettered words. 


\subsection{The Open and Dynamic Principle}

First of all, lettered words should be open and dynamic because it has long been common for a nation to borrow and absorb another nation's language (Gu, 1990). With the globalization of science, technology as well as information in the present world, nations have more frequent communication with each other, hence breaking the time and space domains of culture, recomposing it and promoting mutual interaction. In this way, different cultures of different nations will be able to achieve mutual development by learning from each other. It has been proved that it is helpful for the diversified development of Chinese vocabulary to enhance its expressiveness and capacity. As a newly-born thing, lettered words are not standardized enough yet, but its intermediary position in modern Chinese lexical system shouldn't be neglected but be developed. Certainly, "openness" here doesn't mean all lettered words have access to Chinese or can be used by people (Xue, 2006). During language practice, some lettered words may lose the competition with their corresponding Chinese words while some others may stay as basic parts in modern Chinese lexical system.

Vocabulary is a dynamic and open system by itself (Tian, 2007). Faced with so many lettered words flooding to us, we are supposed to have tolerant attitudes towards them and achieve their normalization through long-time practice. Overall, language normalization should be an open, tolerant, dynamic and developing process, during which modern Chinese may be prevented from development by isolation and conservation.

\subsection{The Principle of "Being Tested in Practice"}

As a dynamically-developed open system, language achieves permanent vitality through constant production of new elements and elimination of old ones. It is generally in the practical use of language that new elements are introduced, competed, normalized and eliminated. Some lettered words may be frequently used by Chinese people after being introduced and then become basic Chinese vocabulary; some may be rejected at the very beginning but are accepted finally; some may be finally eliminated in their competition with other expressions although they were once frequently used. Therefore, language normalization is also a dynamic process in which over-emphasis on standards may lead to stubborn Chinese and too hasty normalization may not be scientific enough because it takes a long time for a lettered word to be tested and accepted finally. Language normalization shouldn't be isolated from language application and development. It is an indispensable principle for language normalization to be based on application and it is a fixed guideline for it to focus on language practice (Zhan, 2001).

\subsection{The Principle of "Being Directed by the Media"}

Loans words find their way to Chinese people's spiritual and material lives through the mass media, such as newspapers, $\mathrm{TV}$, broadcast, Internet and a variety of books and videos, therefore they are of particular importance in providing the right direction for language normalization. Although we are tolerant to loanwords, it doesn't mean leaving it unrestricted at all. As for some people's use of lettered words in order to cater to western culture, the principle of "normalization with proper tolerance" should be emphasized. For example, "copy" isn't quite necessary since we have “复制” and nor is “MTV” with the existence of “音乐电视”. In fact, it is the media's eagerness for quick success that has led to the abuse of pigeon English and lettered words. For example, some media express “举办晚会” as “开 party” in order to appeal to smart young people.

In this case, the government is expected to lay down special regulations to restrict those important propaganda organizations as well as the media in order to encourage them to be serious enough when introducing and using loanwords. With the great leading role of those influential radio stations, TV programs, newspapers and magazines' in using normalized lettered words, a favorable atmosphere will certainly be created. As a result, the use of these words in Chinese will be more sound and efficient and the current unordered use of them will be changed as well. For example, at the very beginning, due to CCTV's use of "MTV" as the title for its “音乐电视” program, “MTV" was quite popular all over China. With it changed into “音乐电视” by Oriental Horizon, other columns in CCTV as well as other local TV stations followed the example, too. So now “音乐电视” seems to be more popular than "MTV".

\subsection{The Principle of "Being Authenticated by Dictionaries"}

Lettered words, arising, depositing and finally disappearing in language practice, can be neither laid down or cancelled by the administration nor normalized by language experts as soon as they appear. What those professionals specialized in language can do is to guide and recommend normalized lettered words by compiling dictionaries or amending instructions based on their close observation, collection, sorting and research of these words. Then, it is up to the public to decide whether to accept them or not after long-term test.

China State Language Commission is expected to have profound investigation, careful analysis and scientific research on loanwords, to thoroughly collect the lettered words commonly used in daily life and science and technology filed, to lay down regulations on the translation and use of lettered words according to the rules of language development, to compile dictionaries of lettered words including exact information, to regularly publicize new lettered words, to normalize the writing, pronunciation and meaning of lettered words, hence providing reasonable and healthy paths for lettered words to enter Chinese lexical system. 87 lettered words are included in Xinhua Dictionary (2001 revised 
edition); 200 ones are included in Compulsory Dictionary of Chinese New Words (2003 edition); 182 ones are included in The Dictionary of Modern Chinese (2005 revised edition). Obviously, authoritative experts and institutions not only emphasize and recognize lettered words but try to normalize them and reasonably utilize them in order to promote the diversification of Chinese lexical system.

\section{Conclusion}

Lettered words in Chinese lexical system are the outcome of information share, scientific and technological interaction and cultural fusion. As one part of Chinese vocabulary, lettered words are also in variation and are to be tested by corresponding standards. Therefore, it should be recognized that it is a long battle to normalize lettered words in dynamic development, hence requiring language researchers, dictionary compilers, the mass media and the authorities to have joint efforts with open, dynamic and developing attitudes. In the normalization process, both the purity and unification of Chinese character system and the diversification of language should be maintained. Therefore, there is no definite limit or standard for it. Instead, it should be accepted that the normalization process is of great vagueness and uncertainty and it tends to be influenced by a variety of factors. Based on that, a normalization pattern involving many factors should be established to instruct practical normalization with different schemes and efforts according to different types (Shi, 2000). To sum up, the elastic principles of "having tolerant attitudes and emphasizing dynamic changes", "being guided by the media and authenticated by dictionaries", "being tested by conventional standards in practice" and "sticking to open development and gradual normalization" should be established.

\section{References}

Gu, Jiazu. (1990). A Discussion of the Absorbing and Assimilative Functions of Language and National Cultural Psychology. Language and Culture. Shanghai: Shanghai Foreign Language Education Press, p41.

Liu, Jianmei. (2002). On the Normalization of Loanwords in Modern Chinese System. Applied Linguistics, (1): p91.

Liu, Yongquan. (2002). On the Issue of Chinese Lettered Words. Applied Linguistics, (1): pp 86-90.

Luo, Changpei. (2004). Cultural Linguistics. Beijing: Beijing Press, p14.

Shi, Youwei. (2000). Chinese Loanwords. The Commercial Press, p14.

Tian, Yuhe. (2007). Several Problems in Researches on Contemporary New Chinese Words. Exploration, (5): pp191-193.

Xue, Xiaocong. (2006). Researches on Lettered Words. Language and Translation, (1): p16.

Zhan, Bohui. (2001). Current Language Phenomena and Language Normalization. Jinan Journal, (7): p126. 